\title{
ACOMPANHAMENTO FARMACOTERAPÊUTICO DE PACIENTES ATENDIDOS EM PRONTO ATENDIMENTO EM UM HOSPITAL DE ENSINO
}

\author{
PHARMACOTHERAPEUTIC MONITORING OF PATIENTS \\ ATENDED IN EMERGENCY CARE IN A TEACHING HOSPITAL
}

\section{Cristiane Carla Dressler Garske', Alice Pereira Freitas', Betina Brixner², Ediberto de Oliveira Machado ${ }^{3}$, Ana Paula Helfer Schneider ${ }^{4}$}

\section{RESUMO}

O objetivo deste estudo foi identificar e quantificar o número de intervenções farmacêuticas realizadas durante o acompanhamento farmacoterapêutico de pacientes internados em unidade de Pronto Atendimento de um Hospital de ensino, de Março a Maio de 2015, através do registro em planilhas eletrônicas realizadas pelos farmacêuticos residentes após avaliação de prescrições médicas. Foram avaliadas 278 prescrições de 190 pacientes, 59\% eram do sexo feminino. A média de idade foi 62,9 $( \pm 17,8)$ anos. Foram realizadas 52 intervenções farmacêuticas, das quais $80,8 \%$ foram aceitas. Através das atividades clínicas busca-se garantir segurança, detectando erros antes que impactem na qualidade da terapia medicamentosa do paciente. A aceitabilidade das intervenções farmacêuticas encontradas indica a necessidade e importância da atuação do farmacêutico clínico no âmbito hospitalar atuando junto à equipe de saúde no cuidado do paciente, tanto no ponto de vista econômico, quanto clínico, garantindo o uso racional de medicamentos, segurança e qualidade do tratamento.

Descritores: Intervenção/Farmacêuticos; Hospitalar.

\section{ABSTRACT}

The aim of this study was to identify and quantify the number of pharmaceutical interventions for pharmacotherapeutic monitoring of patients admitted to the Emergency Unit of a teaching hospital, from March to May 2015, by recording in spreadsheets made by pharmaceutical residents after evaluation of medical prescriptions. 278 prescriptions were evaluated 190 patients, $59 \%$ were female. The mean age was 62.9 ( \pm 17.8 ) years. 52 pharmaceutical interventions, of which $80.8 \%$ were accepted were held. Through clinical activities seek to ensure security by detecting errors before they impact the quality of patient drug therapy. The acceptability of found pharmaceutical interventions indicates the need and importance of the clinical pharmacist activities in hospitals working with the healthcare team in patient care, both in the economic point of view, the clinical, ensuring the rational use of medicines, safety and quality treatment.
${ }^{1}$ Graduada em Farmácia pela Universidade de Santa Cruz do Sul, (UNISC), Santa Cruz do Sul, RS, Brasil.

2 Especialista em Análises Clínicas e Toxicológicas pela Universidade de Santa Cruz do Sul (UNISC), Santa Cruz do Sul, RS, Brasil.

${ }^{3}$ Mestre em Desenvolvimento Regional pela Universidade de Santa Cruz do Sul (UNISC), Santa Cruz do Sul, RS, Brasil.

\footnotetext{
${ }^{4}$ Mestre em PPG Saúde Coletiva pela Universidade do Vale do Rio dos Sinos (UNISINOS), São Leopoldo, RS, Brasil.
}

Descriptors: Intervention/Pharmacists; Hospital. 


\section{Introdução}

O serviço de farmácia clínica, bem como o acompanhamento farmacoterapêutico são de fundamental importância para contribuir com a garantia do acesso, uso racional e segurança dos medicamentos prescritos ${ }^{1}$. A farmácia clínica visa identificar problemas relacionados com medicamentos e as intervenções farmacêuticas, realizadas junto à equipe de saúde, possibilitam uma melhora clínica dos pacientes acompanhados².

O farmacêutico clínico deve realizar orientações sobre os medicamentos - tanto para o paciente, quanto para os profissionais da saúde -, monitorar a farmacoterapia prescrita e intervir, quando necessário, sendo este o principal profissional envolvido no uso de medicamentos e capacitado para avaliar aspectos relacionados a medicamentos, garantindo assim, a segurança do paciente ${ }^{1,3}$. Salienta-se que, não somente o farmacêutico, mas sim todos os profissionais envolvidos com 0 paciente devem estar atentos e seguros no momento da prescrição, dispensação e administração de medicamentos ${ }^{4}$.

De acordo com o Consenso Brasileiro de Atenção Farmacêutica, intervenção farmacêutica é um ato planejado, que deve ser documentado e realizado junto ao usuário e equipe de saúde, que visa prevenir ou resolver problemas que interferem ou podem interferir na farmacoterapia, além disso, a intervenção farmacêutica é parte integrante do processo de acompanhamento farmacoterapêutico ${ }^{5}$.

Grande parte dos erros de medicação é atribuída à equipe de enfermagem por atuar diretamente nas últimas etapas de preparo e administração de medicamentos ${ }^{6}$, no entanto o farmacêutico inserido na equipe assistencial deve detectar estes erros no início ou no meio do sistema, intervir e corrigir possíveis danos ${ }^{7}$.

A análise da prescrição é uma das atividades atribuída ao profissional farmacêutico clínico, intervindo em situações como: reconciliação medicamentosa; forma farmacêutica, via de administração, posologia e administração inadequadas; incompatibilidades entre medicamentos; estabilidade e diluição de medicamentos inadequadas; bem como evitar que o paciente alérgico a determinado medicamento seja prescrito ${ }^{8,9}$.

Com isso, o objetivo do estudo foi identificar e quantificar o número de intervenções farmacêuticas realizadas em pronto atendimento em um hospital de ensino.

\section{Metodologia}

Realizou-se um estudo retrospectivo, com caráter descritivo e transversal, no qual foram quantificadas e analisadas as intervenções realizadas pelos farmacêuticos residentes de um hospital de ensino no interior do Rio Grande do Sul junto à equipe multiprofissional. Trata-se de um hospital de médio porte, referência na região do Vale do Rio Pardo, com serviço de urgência e emergência e intensivismo (neonatal, pediátrico e adulto).

As intervenções encontradas, após as análises das prescrições, foram realizadas a partir de comunicação pessoal com os diferentes profissionais de saúde do hospital ou através das evoluções farmacêuticas registradas nos prontuários dos pacientes. Os dados obtidos de todas as intervenções farmacêuticas realizadas durante o período de internação destes pacientes foram devidamente registrados em planilha eletrônica, que os farmacêuticos residentes preenchem.

Para o presente estudo, foram coletadas as informações registradas no período de março a maio de 2015, obtidas em uma unidade de pronto atendimento do hospital, que possui oito leitos para observação de pacientes graves.

Para este estudo, levou-se em consideração todas as intervenções farmacêuticas realizadas, podendo estas ser via contato oral com médico/equipe de enfermagem e/ou evolução farmacêutica no prontuário do paciente. Variáveis para este estudo, como a aceitabilidade das intervenções, idade e sexo dos pacientes também foram consideradas. Para análise estatística, foi utilizado o software Microsoft Excel 2007 para registrar os dados originais e para a análise exploratória, posteriormente gerando tabelas e gráficos.

A pesquisa foi conduzida de acordo com os padrões éticos exigidos e o projeto foi aprovado pelo Comitê de Ética em Pesquisa da Universidade de Santa Cruz do Sul sob o número 1.252.586/2015. 


\section{Resultados}

Durante os três meses de coleta de dados, foram avaliadas 278 prescrições médicas de 190 pacientes. A predominância dos atendimentos foi à pacientes do sexo feminino $(59 \%)$ com média de idade de $62,9( \pm 17,8)$ anos.

Foram realizadas 52 intervenções farmacêuticas, relacionadas à posologia, reconciliação medicamentosa, estabilidade, substituição de horário de administração do medicamento, diluição incorreta, alergia a medicamentos e troca de forma farmacêutica, conforme demonstrado na Figura 1.

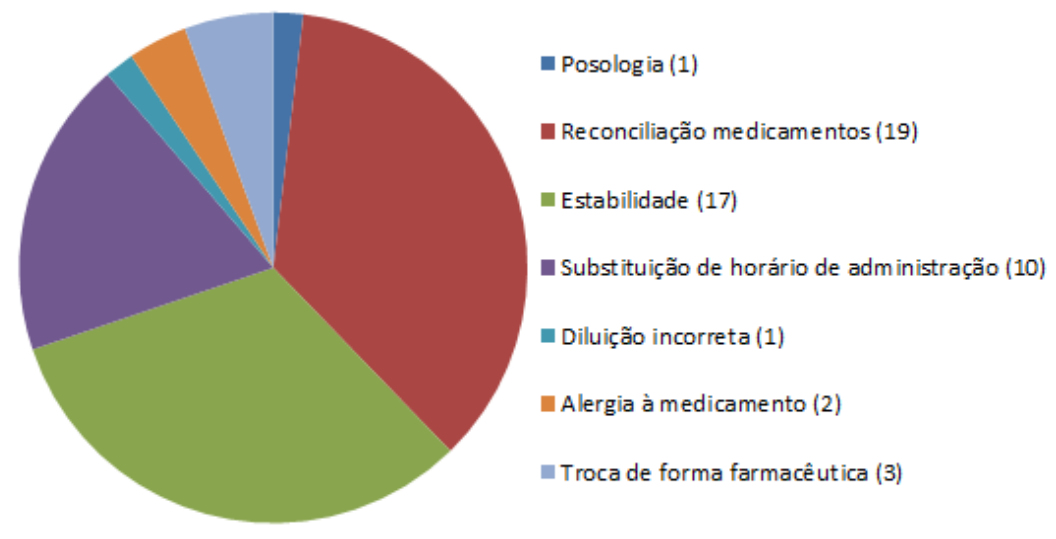

Figura 1 - Intervenções farmacêuticas realizadas nos meses de março a maio de 2015 em unidade de Pronto Atendimento

Das intervenções realizadas referentes à estabilidade medicamentosa, 52,9\% foram relacionadas com a morfina. Quanto à substituição de horário de administração, $60 \%$ destas intervenções foram realizadas para as cápsulas de omeprazol. Na troca de forma farmacêutica, as intervenções ocorreram em pacientes com uso de sonda nasoentérica, onde $66,7 \%$ estavam utilizando omeprazol cápsula, o qual perde sua eficácia quando administrado por esta via.

Do total de intervenções realizadas, 32,7\% foram direcionadas para a equipe de enfermagem e $67,3 \%$ para os médicos prescritores. Quanto à aceitabilidade das mesmas, $80,8 \%$ foram aceitas por ambos profissionais da saúde.

\section{Discussão}

A rotina do acompanhamento farmacoterapêutico prevê que todos os pacientes que fazem uso de medicamentos durante a internação sejam avaliados, onde os farmacêuticos verificam quais medicamentos são utilizados, se tal utilização vem sendo feita de forma correta e se o paciente apresenta histórico de alergias a medicamentos, concordando com o estudo que identifica que ao implementar a farmácia clínica, o profissional farmacêutico se reaproxima do paciente através da interação com este para obter a história de medicamentos de uso contínuo, realizando orientações quanto a dúvidas sobre o uso de medicamentos; além disso, insere-se na equipe interdisciplinar, onde deve assumir a responsabilidade com a farmacoterapia do paciente ${ }^{10}$.

No presente estudo uma intervenção farmacêutica foi relacionada à posologia do medicamento prescrito, destacando-se a importância de administrar a dose adequada, no intervalo correto, evitando sub ou superdosagens e as manifestações dos efeitos adversos ou tóxicos, bem como gastos desnecessários ${ }^{11}$.

No ato de admissão do paciente, informações incompletas podem acarretar em interrupção ou inadequação da terapia medicamentosa, podendo causar problemas à saúde do paciente ${ }^{12}$. Neste estudo $36,5 \%$ das intervenções farmacêuticas encontradas foram relacionadas à reconciliação medicamentosa, que é um processo para obtenção de uma lista completa, precisa e atualizada dos medicamentos que cada paciente utiliza (incluindo nome, dosagem, frequência e via de administração), comparada com as prescrições médicas feitas na admissão, transferência, consultas ambulatoriais e alta hospitalar. Essa lista é usada para aperfeiçoar a utilização dos medicamentos, em todos os pontos de transição do cuidado e tem como principal objetivo diminuir a ocorrência de erros de medicação quando o paciente troca de nível de assistência à saúde ${ }^{13}$. Em estudo realizado em um hospital universitário do Paraná, dos 58 entrevistados, 24 necessitaram de reconciliação medicamentosa e 15 destes apresentavam pelo menos um tipo de erro relacionado ao 
medicamento, sendo necessária a intervenção farmacêutica ${ }^{14}$. No Brasil, os papéis da equipe médica e de enfermagem nas equipes são claros, mas a inclusão de outros profissionais ainda está em andamento. Deve-se considerar que o farmacêutico é especialmente adequado para obter históricos de medicação mais completas e reconciliar discrepâncias devido à sua formação, experiência, familiaridade e conhecimento sobre medicamentos ${ }^{15,16}$. Outros estudos também sinalizam a importância da reconciliação de medicamentos na admissão hospitalar, ao identificarem e corrigirem erros através deste processo ${ }^{17,18}$.

Estando relacionada com a eficácia das medicações, a estabilidade química e física dos medicamentos administrados nos pacientes deve ser respeitada para garantir uma terapia adequada. É necessário levar em consideração a solução reconstituinte, hora do preparo e armazenamento para verificar o prazo de estabilidade das medicações após a sua diluição, garantindo assim uma segurança terapêutica ${ }^{19}$. No presente estudo, 17 intervenções foram relacionadas à estabilidade comprometida das medicações, normalmente pelo preparo antecipado e por doses aplicadas menores do que as preparadas.

Duas intervenções farmacêuticas, deste estudo, evitaram a administração de medicamentos contraindicados para pacientes que apresentavam alergias a medicamentos prescritos. Em um estudo realizado em Fortaleza, foram entrevistadas 957 pessoas em seus domicílios. Destas, 35\% informaram que foram questionadas em sua última consulta, pelo médico no ato da prescrição, quanto a manifestações anteriores de quadros alérgicos a medicamentos ${ }^{20}$. Quando 0 paciente procura um médico, pode ser que ele já tenha apresentado hipersensibilidade a algum medicamento em algum momento de sua vida. Obter essa informação é muito importante para escolha de uma nova terapêutica a fim de evitar expor o paciente a riscos desnecessários ${ }^{21}$.

Neste estudo, foram realizadas três intervenções farmacêuticas relacionadas à troca de forma farmacêutica, devido a esta estar prescrita por via de administração inadequada, podendo obstruir a sonda enteral ou a presença de alimento interagir com o fármaco. Um estudo realizado nos Estados Unidos comprovou que a equipe que consultava o serviço da farmácia estava mais propensa a utilizar as formas farmacêuticas de maneira adequada e relataram menos obstrução nas sondas. Ainda assim, o farmacêutico é pouco solicitado como fonte esclarecedora ${ }^{22}$. Os medicamentos administrados por sonda devem ser adequadamente utilizados para evitar a redução de sua eficácia, desconforto ao paciente, aumento nos efeitos adversos, incompatibilidade dos fármacos e excipientes com a nutrição enteral ou até mesmo com componentes da sonda, obstrução de sondas e aumento dos custos, reforçando assim a importância do farmacêutico clínico.

Das intervenções realizadas neste estudo, $67,3 \%$ foram direcionadas para os médicos prescritores e $32,7 \%$ para a equipe de enfermagem. Quanto a aceitabilidade das mesmas, $80,8 \%$ das intervenções farmacêuticas foram aceitas pela equipe, vindo ao encontro do trabalho realizado em hospital geral de ensino, ao iniciar o serviço de farmácia clínica neste hospital e obter $65 \%$ de aceitabilidade pelos demais profissionais da saúde em suas intervenções farmacêuticas ${ }^{2}$. Dados relacionados das intervenções realizadas junto à equipe de saúde, também foram encontrados no estudo realizado no Instituto Nacional de Traumatologia e Ortopedia, onde as intervenções farmacêuticas de maior incidência foram para a equipe médica $(71,1 \%)$, seguido dos enfermeiros $(16,9 \%)$, digitadores $(6,2 \%)$ e auxiliares de enfermagem $(4,6 \%)$, onde $70 \%$ destas intervenções foram aceitas ${ }^{23}$.

De maneira geral, a equipe apresenta uma boa aceitação quanto às intervenções farmacêuticas, sendo elas, prioritariamente, realizadas com o profissional médico. Além de garantir a segurança, o uso correto e racional dos medicamentos através da análise de prescrições, a farmácia clínica pode contribuir ainda, para a redução de custos ao otimizar a farmacoterapia do paciente, diminuição no tempo de internação, bem como reduzir as taxas de mortalidade ${ }^{24}$. As intervenções e orientações realizadas pelo farmacêutico também impactam na adesão à terapia medicamentosa, sendo esta, mais presente em pacientes que receberam orientações, do que os que não receberam ${ }^{25,26}$.

\section{Considerações Finais}

O diálogo e as decisões de uma equipe multiprofissional são fundamentais para a reconciliação medicamentosa e a redução de potenciais danos aos pacientes. Farmacêuticos, médicos e equipe de enfermagem são indispensáveis na seleção, prescrição, administração de medicamentos, monitoramento e educação dos pacientes durante a internação hospitalar. 


\section{Referências}

1. Bernardi EAT, Rodrigues R, Tomporoski GG, Salvi VM. Implantação da avaliação farmacêutica da prescrição médica e as ações de farmácia clínica em um hospital oncológico do sul do Brasil. Rev Espaço para a Saúde. 2014; 15(2):29-35.

2. Costa JM, Abelha LL, Duque FAT. Experiência de implantação de farmácia clínica em um hospital de ensino. Rev Brasileira de Farmácia. 2013; 94(3): 250-256.

3. Magalhães GF, Santos GBC, Rosa MB, Noblat LAB. Medication Reconciliation in Patients Hospitalized in a Cardiology Unit. PlosOne. 2014; 9(12).

4. Oliboni LS, Camargo AL. Validação da prescrição oncológica: o papel do farmacêutico na prevenção de erros de medicação. Rev do Hospital de Clínicas de Porto Alegre. 2009; 2(29):147-152.

5. Consenso Brasileiro de Atenção Farmacêutica - Proposta. Organização Pan-Americana de Saúde. [online]. 2002 [acesso 2015 Nov 14]. Disponível em: http://bvsms.saude.gov.br/bvs/publicacoes/PropostaConsensoAtenfar.pdf

6. Soerensen AL, Lisby M, Nielsen LP, Mainz J. The medication process in a psychiatric hospital: are errors a potential threat to patient safety? Risk Management and Healthcare Policy. 2013; 6:13-22.

7. Fassarella CS, Bueno AAB, Souza ECC. Segurança do paciente no ambiente hospitalar: os avanços na prevenção de eventos adversos no sistema de medicação. Rede de Cuidados em Saúde Rio de Janeiro. 2013;7(1).

8. Miranda TMM, Petriccione S, Ferracini FT, Filho WMB. Intervenções realizadas pelo farmacêutico clínico na unidade de primeiro atendimento. Einstein, 2012;10(1):74-78.

9. Francis J, Abraham S. Clinical pharmacists: bridging the gap between patients and physicians. Saudi Pharmaceutical Journal. 2014; 22(6):600-602.

10. Almeida RB, Mendes DHC, Dalpizzol PA. Ensino farmacêutico no Brasil na perspectiva de uma formação clínica. Revista de Ciências Farmacêuticas Básica e Aplicada. 2014; 35(3):347-354.

11. Bontempo VL, Coelho MC, Chaves JG, Pereira LAM, Andrade WW, Braccini CM. Análise das prescrições geradas pelas equipes do PSF e das unidades de atendimento imediato, em dois distritos sanitários do município de Contagem/ MG. Bol Pneumol Sanit. 2003;11(2):17-24.

12. Cornish PL, Knowles SR, Marchesano R, Tam V, Shadowitz S, Juurlink DN, Etchells EE. Unintended medication discrepancies at the time of hospital admission. Arch Intern Med. 2005;165:424-429.

13. Anacleto TA, Rosa MB, Neiva HM, Martins MAP. Erros de medicação - Farmácia hospitalar. Pharm. Bras. [online]. 2010 [acesso 2015 Nov 19]. Disponível em: http://www.cff.org.br/sistemas/geral/revista/pdf/124/encarte_farmaciahospitalar.pdf 14. Frizon F, Santos AH, Caldeira LF, Menolli PVS. Reconciliação de medicamentos em hospital universitário. Rev enferm UERJ. 2014;22(4):454-460.

15. Galvin M, Jago-Byrne MC, Fitzsimons M, Grimes T. Clinical pharmacist's contribution to medication reconciliation on admission to hospital in Ireland. Int J Clin Pharm. 2013;35:14-21.

16. Schnipper JL, Kirwin JL, Cotugno MC, Wahlstrom SA, Brown BA, Kachalia A, Horng M, Roy CL, Mckean SC, Bates DW. Role of pharmacist counseling in preventing adverse drug events after hospitalization. Arch Intern Med. 2006;166:565-571.

17. Unroe KT, Pfeiffenberger T, Riegelhaupt S, Jastrzembski J, Lokhnygina Y, Colón-Emeric C. Inpatient medication reconciliation at admission and discharge: A retrospective cohort study of age and other risk factors for medication discrepancies. Am J Geriatr Pharmacother. 2010;8(2):115-126.

18. Schuch AZ, Zuckermann J, Santos MEF, Martinbiancho JK, Mahmud SDP. Reconciliação de medicamentos na admissão em uma unidade de oncologia pediátrica. Rev. Bras. Farm. Hosp. Serv. Saúde São Paulo. 2013;4(2):35-39.

19. Camerini FG, Silva LD. Segurança do paciente: análise do preparo de medicações intravenosa em hospital da rede sentinela. Texto Contexto Enferm. 2011;20(1):41-49.

20. Arrais PSD, Barreto ML, Coelho HLL. Aspectos dos processos de prescrição e dispensação de medicamentos na percepção do paciente: estudo de base populacional em Fortaleza, Ceará, Brasil. Cad. Saúde Pública. 2007;23(4):927-937. 21. Mosegui GBG, Rozenfeld S, Veras RP, Vianna CMM. Avaliação da qualidade do uso de medicamentos em idosos. Rev Saúde Pública. 1999;33:437-444.

22. Thomson R. Heallthcare Series: Micromedex, Greenwood Village, Colorado. [online] 2015. [acesso 2015 Nov 21]. Disponível em: http://www.micromedex.com/

23. Nunes PHC, Pereira BMG, Nominato JCS, Albuquerque EM, Silva LFN, Castro IRS, Castilho SR. Intervenção farmacêutica e prevenção de eventos adversos. Revista Brasileira de Ciências Farmacêutica. 2008;44:691-699. 
24. Pinto IVL, Castro MS, Reis AMM. Descrição da atuação do farmacêutico em equipe multiprofissional com ênfase no cuidado ao idoso hospitalizado. Revista Brasileira de Geriatria e Gerontologia. 2013;16(4):747-458.

25. Hatah E, Braund R, Tordoff J, Dufful SB. A systematic review and meta-analysis of pharmacist-led fee-for services medication review. British Journal of Clinical Pharmacology. 2013;77(1);102-115.

26. Kuhmmer R, Lima KM, Ribeiro RA, Hammes LS, Bastos GAN, Souza MCSC, Polanczyk CA, Rollin GAFS, Caon S, Guterres CM, Leite EA. Effectiveness of pharmaceutical care at discharge in the emergency department: study protocol of a randomized controlled trial. Trials. 2015;60(16).

\section{Cristiane Carla Dressler Garske}

Endereço para correspondência - Rua: Manaus, $n^{\circ} 685$, ap 219, Bloco $E$ Bairro: Vila Schulz, CEP: 96845500, Santa Cruz do Sul, RS, Brasil.

E-mail: crisdressler2005@yahoo.com.br

Lattes: http://lattes.cnpq.br/7469653524983533

Alice Pereira Freitas - alicefreit@hotmail.com

Betina Brixner - betinabrixner@yahoo.com.br

Ediberto de Oliveira Machado - emachado@unisc.br

Ana Paula Helfer Schneider - anahelfer@unisc.br

\section{Enviado em 20 de janeiro de 2016. Aceito em 18 de março de 2016.}

